\title{
Examining the information and communication technologies enabling servitized manufacture
}

\begin{abstract}
Services-led competitive strategies are critically important to western manufacturers. This paper contributes to our foundational knowledge of such strategies by examining the enabling information and communication technologies that successfully servitized manufacturers appear to be adopting. Although these are preliminary findings from a longer-term research programme, through this article we seek to offer immediate assistance to manufacturers who wish to understand how they might exploit the servitization movement.
\end{abstract}

\section{Introduction}

Manufacturers have found themselves in a position where price is the key differentiator in global markets, even for those companies producing high value, technologically complex products such as aero engines, locomotives, agricultural machinery and machine tools. Consequently, many manufacturers have broadened their focus from production to include advanced services. These integrated product-service offerings can be distinctive, long-lived, and easier to defend from competition from lower cost economies (Baines et al, 2009). Therefore, it comes as little surprise that service provision is now a conscious and explicit strategy for market differentiation and ultimately competitive advantage. This is referred to as servitization or rather the infusion of services into manufacturing.

These advanced product-centric services frequently require the original equipment manufacturer (OEM) to take on a greater risk for asset performance, availability and reliability. Such pressures have driven manufacturers to develop technological systems that improve their visibility of assets in the field. These systems combine hardware (for example, sensor and wireless technologies) and software (for example, signal processing and analysis techniques) to identify current and predicted 'health' of an asset. This technology is a critically important enabler of advanced product - centric services. However, little is known about the generic structure of such systems and exactly how their capabilities are integrated into the more conventional information systems of manufacturers. Therefore, this paper contributes to our knowledge of servitization by examining information and communication technologies (ICTs) that support the delivery of advanced services. To realise this purpose we have organised this paper to first summarise the scope of information systems in conventional production-centric operations, then summarise our research design, before presenting the findings from this study.

\section{Information and communication technologies within conventional production operations}

Within conventional production operations any ICT capabilities that exist tend to be focused at the planning and control of production. This is a common theme within much of the literature associated with manufacturing system design and the most popular systems are Manufacturing Resource Planning (MRPII) and Enterprise Resource Planning (ERP). Both are integrated hardware and software applications linked to a central database that stores and delivers business data and information. MRPII is concerned with the coordination of the entire manufacturing production, including materials, finance, and human relations. Such a system facilitates the development of a 
detailed production schedule based around machine and labour capacity, and scheduling of production runs according to the arrival of materials. A typical MRPII output is a final labour and machine schedule, along with data about the cost of production, including machine time, labour time and materials used, as well as final production numbers. Enterprise Resource Planning (ERP) can be thought of as an extension of MRPII and sets out to manage internal and external resources. Its purpose is to facilitate the flow of information between all business functions inside the boundaries of the organization and manage the connections to external stakeholders. However, connections to the asset in the field are limited, and largely consist of customer relationship databases with records of ownership and history. Conventionally, very little data is captured about equipment condition and how it is used by customers in the field.

\section{Research design summary}

Our purpose has been to understand the differences between the information and communication technologies used in production operations and those used by successful servitized manufacturers. In short, our method has been to carry out in-depth and multi-disciplinary case-studies of leading manufacturers in delivery of advanced product-centric services. Our partner organisations for this study have been Caterpillar, Alstom, MAN and Xerox. Our approach has been to carryout semistructured interviews with personnel ranging from Customer Services, and Service Marketing, through to Chief Executive Officers. Overall some 140 hours of interviews were conducted. In each case the focus of the interviews has been on the generic structure of the ICTs that are used to support advanced product-centric services, along with understanding the factors that have led to their construction. Typical advanced product-centric services include risk and revenue sharing, payper-use, support agreements and availability contracts. These results were then analysed to establish the findings from this study.

\section{Information and communication technologies enabling servitized operations}

Competitive pressures stimulate innovations within manufacturing operations. Therefore, prior to examining the information and communication technologies that servitized manufacturers have developed, it is first necessary to explain the pressures that are particular to delivering advanced services contracts. In this section we first summarise these, then present the generic configuration apparent across the manufacturers in our study, and then present the rational that explain their adoption.

Underlying business pressures driving the establishment of enabling technology and systems: Our case work has revealed that where a manufacturer sets out to adopt advanced services two sets of pressures incurred. One reflects the contractual obligations from the customer and is typically measured in terms of performance, availability and reliability. Here, performance is concerned with the extent to which the full capability of equipment is accessible, for example the power delivered by a gas-turbine as a percentage of that specified. Availability is usually assessed as the extent of time that a product or asset is available for use as a proportion of the scheduled availability. Reliability is taken as a measure of mean-time between in-service failures and again the capability to monitor the asset in use and to analyse and record operational performance issues enables feedback into the asset design loop to improve its designed reliability. The second principle pressure is from the host manufacturer, and is concerned with the resources required to deliver the service offering, and is typically measured as contract delivery cost. These pressures to deliver asset performance, 
availability and reliability, with the minimum of cost to the host organisation. These pressures help to explain the emergence of ICTs by manufacturers, and the form that such systems typically take can now be explained

The emergent technology and systems: Information systems and management processes (Brax, 2005), along with tools to allow enhancement of company knowledge and best practice (Byron, 2006), and are increasingly seen as essential to competitive success. In conventional manufacture the tendency is towards systems that control the flow of materials through production operations. However, to deliver advanced product-centric services these systems are different. In principle they appear as:

'The use of Information and Communication Technologies (ICTs) to monitor assets remotely and coupled with analysis to inform and advance actions'

Figure 1 illustrates the configuration of ICTs typically used by manufacturers in the efficient and effective delivery of advanced product-centric services.

Figure 1: Illustrating the relationship between the enabling technologies

At a general level the process commences with the real-time monitoring of critical systems and subsystems, periodic transmission of raw and/or analysed data or fault codes via an appropriate communications medium (Satellite, Cellular, GPRS), which then arrives at a control centre where the data can be automatically stored, retrieved and then analysed. Such analysis is typically performed using algorithms and / or experts in condition monitoring and characteristics of the equipment. Manual observation and customer feedback also forms part of the information set at the control centre. This information set is used to generate advanced warning of potential problems and enables the scheduling of materials and/or resources to undertake any necessary maintenance / repair activities implement contingency actions, inform the customer and also feedback to the product design process if needed. 
As an example of this, the engine management system in MAN (UK, Truck \& Bus) vehicles is connected using the vehicle's CANbus to a data acquisition device that allows the continuous collection of information concerning the operation and performance of the vehicle. This data, including GPS location, is transmitted in real-time to the MAN U.K. control centre using cellular communications. The data collected can be used to provide reports showing how the vehicle is being driven together with the actual fuel consumption and whether optimum fuel efficiency is being achieved. Vehicles and drivers can be compared by vehicle type, depot, route or other parameters. Also Alstom (TLS) provides Virgin Trains UK West Coast Mainline fleet with a maintenance, repair and overhaul capability against an availability contract. To effectively and efficiently deliver this service Alstom has introduced a range of enabling technology solutions to provide dynamic data on train status and location underpinned by an on-board Train Management System (TMS) that monitors a range of 25 train systems that include propulsion, tilt, high tension, braking, air etc. using around 15 of computers interfaced to various on-board sensors. Using GPRS the TMS sends fault codes to fleet control centres staffed by 'experts', who communicate with the maintenance depots the work needed, including parts, to rectify faults identified prior to the arrival of the train at maintenance depots where routine maintenance and fault rectification has to be completed in a narrow time. The TMS data, 'expert' analysis data and work scheduling and parts acquisition is integrated using a sophisticated ERP system.

Underlying relationship between business pressures and ICT capabilities: The relationship between the enabling ICT and business pressures is captured in figure 2. This illustrates that there are five principal routes through which these capabilities benefit the host manufacturer.

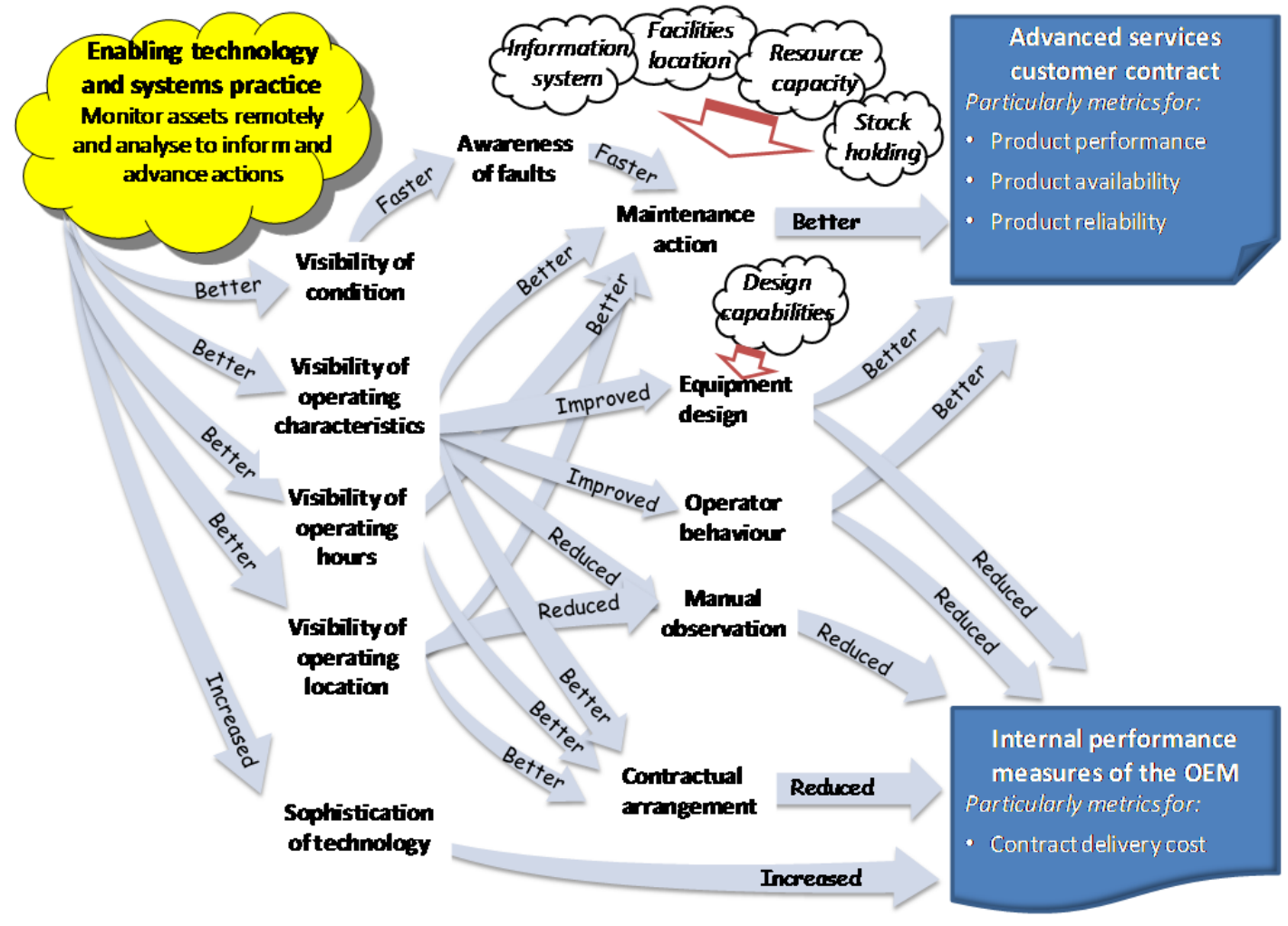

Figure 2: Relationship between ICT capabilities and key performance measures for advanced services contracts 
The enabling technology and systems practices provide better visibility of the asset in use in terms of condition, operating characteristics, time in use and location. The remote access to this information for the service provider facilitates more timely awareness of faults to provide faster maintenance / repair actions and can lead to improved equipment design, operator behaviour and less need for manual observation of the asset in the field. The resulting improved responsiveness of the service provider has a positive impact on asset performance and availability, whilst the improvements in design and operator behaviour have a positive impact on reliability.

The increasing sophistication of enabling technologies and systems has a downside in that it contributes to an increase in service delivery costs. However, the visibility provided of asset operating characteristics, time in use and location can help to mitigate some of the risks adopted by the service provider and relieve contract delivery costs. Also improvements in product reliability and availability can reduce service delivery cost, and can reduce need for manual observation / intervention within the delivery system.

These enabling technology and systems practices, and their implications on business performance, are moderated by other practices within the broader service delivery system. Key here is design and technical capabilities together with integrated information systems. As illustrated in figure 1, decisions about these other practices impact the consequences of the technology and systems practices in a number of ways. For example, design and technical capability and resources are essential to an effective problem response process, as is the ability to integrate information systems to plan materials and resources in a timely and accurate manner.

\section{Concluding remarks and future research}

Our research indicates that ICT capabilities adopted by successfully servitizing manufactures can differ significantly to those in conventional production operations. Fundamentally this is because the business pressures and subsequent performance measures also differ. Production tends to focus on cost, quality and delivery, where as advanced services contracts centre on performance, availability, reliability and cost. These demand that a manufacturer is responsive, and to achieve this they have to ensure that they have visibility of condition and operation of the asset in use by the customer. Again, these decisions about technology and systems practices have to be considered within the context of the wider service delivery system. Our future work will therefore set out to understand some of these other factors in greater detail, and we will report on these in the near future.

\section{References}

Baines, T.S, Lightfoot, H., Peppard, J., Johnson, M., Tiwari, A., Shehab, E., and Swink, M. (2009), "Towards an operations strategy for product-centric servitization", International Journal of Operations and Production Management, 29, 5, 494-519.

Brax S., (2005), "A manufacturer becoming service provider - challenges and a paradox", Manufacturing Service Quality, Vol. 15, No. 2, pp. 142 - 156.

Byeron A. et al, (2006), "The right service strategies for product companies", McKinsey Quarterly, Vol. 1, pp. 40-51 